\title{
RIGHT ANNIHILATOR ALGEBRAS
}

\author{
M. F. SMILEY
}

1. Introduction. The purpose of this note is to extend to arbitrary Banach spaces the characterization given by Bonsall and Goldie $[1 ; 2]$ of the Banach algebra $F(X)$ of all approximately finite-valued operators on a reflexive Banach space $X$. This extension was suggested by one of the results of Bonsall and Goldie [2, Theorem 15]. The requirements on a topologically simple Banach algebra $A$ in order that it be isometric and isomorphic to some $F(X)$ are that every left ideal which is not dense in $A$ has a nonzero right annihilator and a certain restriction on the spectral radius of some nonzero multiple of every element of $A$. (See Condition (3).) Our arguments are merely refinements and simplifications of those of Bonsall and Goldie. Although we have made some effort to make our one-handed presentation fairly self-contained, the reader is assumed to have a knowledge of the arguments employed by Bonsall and Goldie.

We devote $\$ 1$ to the reduction of our problem (in a slightly more general setting) to the topologically simple case. In $\$ 2$ we offer refinements of some of the arguments of Bonsall and Goldie which lead to the desired characterization.

As to notation, we let $E_{r}\left(E_{l}\right)$ denote, for a subset of a ring $A$, all those $x$ in $A$ for which $E x=0(x E=0)$. If $F$ is also a subset of $A$, $E F$ denotes the totality of products $x y$ with $x$ in $E$ and $y$ in $F$, while $E \cdot F$ denotes the additive subgroup of $A$ generated by $E F$. When $A$ is a topological ring, $E^{-}$denotes the closure of $E$. We call $E$ modular in case $A(1-f) \subset E$ for some $f$ in $A$. None of this notation or terminology is new.

2. Semi-simple right annihilator rings. We shall call a topological ring $A$ a right annihilator ring in case

(1) If $I$ is a left ideal of $A$ and $I^{-} \neq A$, then $I_{r} \neq 0$,

(2) Each modular maximal left ideal of $A$ is closed.

Observe that it is a consequence of (1) and (2) that $I_{r} \neq 0$ for each proper modular left ideal $I$ of $A$ because we may imbed $I$ in a modular maximal left ideal and apply (2) and (1). We shall assume throughout this section that $A$ is semi-simple.

If $a$ in $A$ is not left quasi-regular (l.q.r.), then $A(1-a)$ is a proper modular left ideal and hence $(A(1-a))_{r}=[1-a]_{r} \neq 0$, or $a b=b \neq 0$ for some $b$ in $A$. Now let $M$ be a modular maximal left ideal of $A$ so

Received by the editors December 28, 1954. 
that $M_{r} \neq 00$. Not every element in $M_{r}$ is 1.q.r., because if every element of $M_{r} A$ is l.q.r. so is every element of $A M_{r}$ [4, p. 154], $M_{r}$ is contained in the radical of $A, M_{r}=0$, a contradiction. Let $e$ in $M_{r}$ not be 1.q.r. so that $e b=b \neq 0$ for some $b$ in $A$. Then $e$ is not in $M+A(1-e)$, for $e=m+x-x e$ with $m$ in $M$ and $x$ in $A$ yields $e b=0$ $=b$, a contradiction. Since $M$ is maximal, $A(1-e) \subset M, A(1-e) e=0$, $e=e^{2}$ is idempotent in $M_{r}$. Then $M \subset[a]_{l}=A(1-e)$ gives $M=A(1-e)$, $M_{r}=e A$. Since $M$ is maximal, $A e$ is minimal and so is $e A[5, \mathrm{p} .13]$. Because $\left(M_{r}\right)_{l} \supset M$ and is maximal $\left[5\right.$, p. 13], we obtain $\left(M_{r}\right)_{l}=M$. Thus one of the dual rules of Kaplansky [4] holds for modular maximal left ideals of $A$. It is not known whether this rule holds for all closed left ideals of $A$ (cf. [2]).

If $J$ is a left ideal of $A$ and $J^{-} \neq A$, then $J_{r}$ contains a minimal right ideal. For, $J_{r}$ contains an element $a$ which is not 1.q.r. and we have $a b=b \neq 0$ for some $b$ in $A$. Then, as in the previous paragraph, $a$ is not in $J+A(1-a)$ and there is a modular maximal left ideal $M$ such that $M \supset J+A(1-a)$. Then $M_{r}$ is a minimal right ideal and $M_{r} \subset J_{r}$. We apply this to the join $J$ of all the minimal left ideals of $A$ and obtain $J^{-}=A$, for otherwise $J_{r}$ would contain a minimal right ideal whose idempotent generator would have to be in $J$, a contradiction. If $I$ is a minimal left ideal of $A$, then the closure of the two-sided ideal of $A$ generated by $I,\{I\}$, is a minimal closed two-sided ideal of $A$, and every minimal closed two-sided ideal $B$ of $A$ has this form. For, if $B \cap I \neq 0$, then $I \subset B,\{I\}=B$, while $B \cap I=0$ for every minimal left ideal $I$ of $A$ yields $b J=0, b A=0, b=0$ for every $b$ in $B$, a contradiction. Thus $A$ is the closure of the join of its minimal closed two-sided ideals, and this join is clearly direct.

Now let $B$ be one of the minimal closed two-sided ideals of $A$ and let $I$ be a left ideal of $B$ such that $K=I^{-} \neq B$. Then $K$ is a left ideal of $A$, and so is $L=K+B_{l}$. (This proves that $B$ is topologically simple.) If $L^{-}=A$, and $b, b^{\prime} \in B$, then every neighborhood of $b$ contains $k+c$ with $k$ in $K$ and $c$ in $B_{l}$. If $W$ is a neighborhood of $b^{\prime} b$, then $b^{\prime} Z \subset W$ for some neighborhood $Z$ of $b$ and hence $b^{\prime} k \in W, b^{\prime} b \in K^{-}=K$. But then $(B \cdot B)-C K, B \subset K$, a contradiction. Thus $L^{-} \neq A$ and $L_{r}$ contains a minimal right ideal $e A$. If $e$ is not in $B$, then $B e=0, e \in B_{r}=B_{l}$ (because $B_{r} B \subset B \cap B_{r}=0, B_{r} \subset B_{l}$, and dually), $e \in L, e=0$, a contradiction. Hence $e \in B$ and $L e=K e=0, K_{r} \cap B \neq 0$. Finally, let $N$ be a modular maximal left ideal of $B$ so that $B(1-f) \subset N$ for some $f$ in $B$ and hence $f$ not in $N$. Then $f$ is not in $N+A(1-f)$ since $f=n+a-a f$ with $a$ in $A$ and $n$ in $N$ yields $f^{2}=f n+f a-f a f$ in $N$, which with $f-f^{2}$ in $N$ gives $f$ in $N$, a contradiction. Thus there is a modular maximal left ideal $M$ of $A$ such that $M \supset N+A(1-f)$ and hence $f$ is not in 
$M=M^{-}$. Suppose that $f \in N^{-}$, then $f^{2} \in A N^{-} \subset A M^{-}=A M \subset M$, $f-f^{2} \in M, f \in M$, a contradiction. Since $N$ is maximal in $B$ and $B$ is closed, it follows that $N$ is closed.

When we recall that every ideal $B$ of a semisimple ring $A$ is also semisimple (Direct proof: If $b \in B$ and $b c$ is r.q.r. for every $c$ in $B$, then $b a b a$ is r.q.r. for every $a$ in $A$ and thus $b a$ is r.q.r. for every $a$ in $A, b=0$ ), we may summarize the results of this section in a theorem.

THEOREM 1. If $A$ is a semi-simple topological ring which satisfies conditions (1) and (2), then $A$ is the closure of the direct join of its minimal closed two-sided ideals, each of which is a semi-simple and topologically simple ring which satisfies (1) and (2).

3. Topologically simple right annihilator algebras. We now impose, in addition to the assumptions of $\S 1$, the requirements that $A$ is a Banach algebra and that $A$ is topologically simple. (Of course, condition (2) is now redundant.) If $e A$ is a minimal right ideal of $A$, with $e$ idempotent, and $x R_{a}=x a$ for $x$ in $e A$, then $a \rightarrow R_{a}$ is a continuous isomorphism of $A$ onto the ring $R_{A}$, provided we employ the uniform topology for operators in the Banach space $X=e A$. Bonsall and Goldie show, without using the dual of condition (1), that $R_{A}$ includes all finite-valued operators on $X[2$, Theorem 10]. It does not seem to be known whether $R_{A}$ is necessarily closed. However, $\|a\|_{1}=\left\|R_{a}\right\|$ yields a second norm for $A$ such that $\|a\|_{1} \leqq\|a\|$ for every $a$ in $A$. In order to secure a faithful representation of $A$, we impose the following generalization of the requirement of Bonsall [1, Equation (2.1)].

(3) If $a \in A$ with $\|a\|=1$ and $0<\epsilon<1$, then for some $a^{\#}$ in $A$ with $\left\|a^{\sharp}\right\|=1$, we have $\left\|\left(a a^{\sharp}\right)^{n}\right\| \geqq(1-\epsilon)^{n}$ for every positive integer $n$.

That (3) holds in $F(X)$ may be seen by a trivial modification of the proof of Theorem 1 of [1]. It is also clear that if (3) holds for a family of Banach algebras, it also holds in the completion of their direct join ( $=B(\infty)$ sum). When $A$ is not topologically simple, the condition (3) for $A$ implies that each minimal closed two-sided ideal $B$ of $A$ satisfies condition (3). For consider $b \in B$ with $\|b\|=1$ and $0<\epsilon<1$. With $\delta=1-(1-\epsilon)^{1 / 2}$, we obtain $a^{\#}$ in $A$ with $\left\|a^{\sharp}\right\|=1$ and $\left\|\left(b a^{\#} b a^{\sharp}\right)^{n}\right\|$ $\geqq(1-\epsilon)^{n}$ for every $n$. We note that $1 \geqq\left\|a^{\#} b a^{\sharp}\right\| \geqq\left\|b a^{\#} b a^{\sharp}\right\| \geqq 1-\epsilon>0$, and we may set $b^{*}=a^{\#} b a^{*} /\left\|a^{*} b a^{\#}\right\|$ in $B$ to find that $\left\|\left(b b^{*}\right)^{n}\right\| \geqq(1-\epsilon)^{n}$, as desired. (One should observe that an analogous argument applies in the $B^{\#}$ algebras of Bonsall.)

Let us return to the topologically simple case and use condition (3). Following Bonsall, we let $A_{1}$ be the completion of $A$ relative to $\|a\|_{1}$ and prove that the spectral radius, $r(a)$ of $a$ in $A$ is equal to the 
spectral radius, $r_{1}(a)$, of $a$ in $A_{1}$. It is sufficient to observe that it is impossible that an element $b$ of $A$ be q.r. in $A_{1}$ and not q.r. in $A$. For, if $b$ is l.q.r. in $A$, it is also r.q.r. in $A$ by the uniqueness of the quasi-inverse in $A_{1}$. But when $b$ is not l.q.r., $b c=c \neq 0$ for some $c$ in $A$ and $b$ cannot be q.r. in $A_{1}$. Next we prove that $\|a\|=\|a\|_{1}$ for every $a$ in $A$. For suppose that $\|a\|_{1}=1-\delta$ with $\|a\|=1$ and $0<\delta<1$, then with $\epsilon=\delta / 2$, we obtain $a^{\#}$ in $A$ with $\left\|a^{\#}\right\|=1$ and $\left\|\left(a a^{\#}\right)^{n}\right\| \geqq(1-\epsilon)^{n}$. Then we have $(1-\delta)\left\|a^{\sharp}\right\|_{1} \geqq\left\|a a^{\sharp}\right\|_{1} \geqq r_{1}\left(a a^{*}\right)=r\left(a a^{\#}\right) \geqq \lim \left\|\left(a a^{*}\right)^{n}\right\| 1 / n$ $\geqq 1-\epsilon$. Since $1-\epsilon>1-\delta$, this yields $\left\|a^{*}\right\|_{1}>\left\|a^{*}\right\|=1$, a contradiction. Thus $\|a\|_{1}=\|a\|$ for every $a$ in $A$ and the mapping $a \rightarrow R_{a}$ is an isometric isomorphism. We note that (3) implies the semi-simplicity of $A$ (cf. [1, Theorem 4]) and conclude with the promised characterization.

Theorem 2. If a Banach algebra $A$ satisfies the conditions (1) and (3), then $A$ is the completion of the direct join of its minimal closed twosided ideals, each of which is a Banach algebra which satisfies (1) and (3) and is isomorphic and isometric to $F(X)$ for some Banach space $X$. Conversely, $F(X)$ is a topologically simple Banach algebra which satisfies (1) and (3).

\section{REFERENCES}

1. F. F. Bonsall, $A$ minimal property of the norm in some Banach algebras, J. London Math. Soc. vol. 29 (1954) pp. 156-164.

2. F. F. Bonsall and A. W. Goldie, Annihilator algebras, Proc. London Math. Soc. (3) vol. 4 (1954) pp. 154-167.

3. I. Kaplansky, Dual rings, Ann. of Math. vol. 49 (1948) pp. 689-701.

4. - Topological rings, Amer. J. Math. vol. 69 (1947) pp. 153-183.

5. N. Jacobson, On the theory of primitive rings, Ann. of Math. vol. 48 (1947) pp. 8-21.

The State University of Iowa 